\title{
BIBLIOGRAPHY.
}

I. Leber.-Arch. f. Ophthal., Vol. 81, p. I, 1912.

2. Leber.-Graefe-Saemisch Handbuch f. Augenkeilk., " Die Krankheit der Netzhaut," .p. 28.

3. Coats.-Roy. Lond. Ophthal. Hosp. Rep., Vol. 17, p. 449.

4. Hata, B. - Annual Meeting Ophthal. Soc. of Japan, 1919.

\section{DECENTRATION AND OBLIQUE CYLINDERS}

BY

\author{
A. S. Percival, M.A., M.B.(Camb.) \\ NEWCASTLE-UPON-TYNE
}

WHEN prescribing spectacles it is frequently necessary to give a correction for a hyperexo- or a hypereso-phoria, and there are three ways of doing this.

The simplest, and the worst, way is to correct the hyperphoria in one eye with a vertical prism, and to correct the lateral defect in the other eye with a lateral prism. Suppose for instance that a patient needs a correction of $2 \nabla$ for a right hyperphoria and a correction of $3.5 \nabla$ for an exophoria ; he might be given a $2 \nabla$ prism edge up before his right eye, and a $3.5 \nabla$ prism edge out before his left eye.

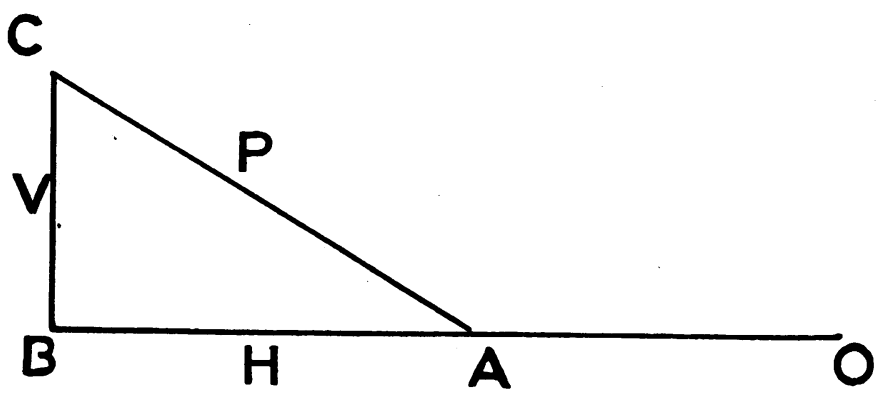

FIG. 1.

The second method is to divide the necessary correction between the two eyes. As is well known, prisms obey the parallelogram law, i.e., a prism set obliquely at an angle $a$ with the horizontal may be resolved into two components:-(Fig. 1.)

$\mathrm{V}$ the vertical component, where $\mathrm{V}=\mathrm{P} \sin \alpha$.

$\mathrm{H}$ the horizontal component, where $\mathrm{H}=\mathrm{P} \cos \alpha$.

$\mathrm{We}$ are given $\mathrm{V}$ and $\mathrm{H}$, and we have to find $\mathrm{P}$ and $\alpha$. Of course $\mathrm{P}=\sqrt{ }-\overline{\mathrm{V}^{2}+\mathrm{H}^{2}}$, and $\tan \alpha=\frac{\mathrm{V}}{\mathrm{H}}$

A simple and sufficiently accurate way of doing this is to draw $\mathrm{AB}$ horizontal representing in direction and units of length 
(centimetres) the power and action of the horizontal component $\mathrm{H}$, and from $\mathrm{B}$ to draw a perpendicular $\mathrm{BC}$ to similarly represent $\mathrm{V}$; then $\mathrm{AC}$ is the vector representing in length and direction the power and the inclination of the equivalent prism $P$. In this case as a $1 \nabla$ vertical prism and a $1.75 \nabla$ lateral prism has to be provided for each eye, draw $A B 1.75 \mathrm{~cm}$. long (A representing the base of the prism) and erect the perpendicular $B C 1 \mathrm{~cm}$. in height ( $\mathrm{B}$ representing the base of the vertical prism); join $\mathrm{AC}$, then $\mathrm{AC}$ which is $2.0155 \mathrm{~cm}$. long represents the required prism for the right eye with its edge pointing outwards and upwards at the angle $\alpha$. One would order a $2 \nabla$ prism pointing outwards and upwards at the angle $30^{\circ}$ for the right eye, and a similar prism for the left eye pointing outwards and downwards, $30^{\circ}$. The $2 \nabla$ correction for the hyperphoria would then be given, and $3.446 \nabla$ instead of $3.5 \nabla$ for the exophoria. It will be noticed that now the two prisms together only amount to $4 \nabla$, whereas according to the first method the sum of the two prisms is $5 \cdot 5 \nabla$.

There is a third way of dealing with the problem if spherical or spherocylindrical lenses are to be worn, which is much easier for the optician and much cheaper for the patient. Suppose that the patient is a myope of $-5 \mathrm{D}$; we know that if $\mathrm{N}$ denote the number of centrads (or prism dioptres) required, and if $l$ denote the decentration in millimetres, $l=\frac{10 \mathrm{~N}}{\mathrm{D}}$.

In this case $\mathrm{N}=2$, and $\mathrm{D}=-5$

$$
\text { so } l=\frac{20}{-5}=-4 \mathrm{~mm} \text {. }
$$

The minus sign reminds one that the decentration must occur in the direction of the thin edge of the prism, so the right lens must be decentred outwards and upwards $30^{\circ}$ along the line A C (Fig 1), and the left lens outwards and downwards $30^{\circ}$. If the decentration had carried a positive sign, i.e. if the lens had been convex, the decentration would have given the direction of the base of the prism, as the centre is always the thickest part of a convex lens.

All this is well known, and was practically pointed out by Mr. Charles Prentice, of New York, in 1890 ; but should the patient have oblique astigmatism, the matter is not so simple, for cylindrical lenses do not obey the parallelogram law.

\section{Oblique Cylinders}

A cylindrical lens of $C$ dioptres set with its plane axis at $0^{\circ}$ exerts that power only in the $90^{\circ}$ plane, it exerts no power in the $0^{\circ}$ plane and its power in an intermediate plane $\theta$, which may be denoted by $\mathrm{C}_{\theta}$ must be determined.

Now every oblique section of a cylinder is an ellipse; let $\mathrm{ABA}^{\prime}$ 
represent the elliptical contour of the oblique section of a cylindrical lens (Fig. 2) the angle of obliquity being $\theta$ or OCA.

$$
\begin{gathered}
\frac{\mathrm{OA}}{\mathrm{CA}}=\sin \theta \text {, but } \mathrm{OA}=\mathrm{CB} \text { or } \mathrm{b} \text {, and } \mathrm{CA}=\mathrm{a} \\
\cdot \cdot \frac{\mathrm{b}}{\mathrm{a}}=\sin \theta \text {, or } \mathrm{a}=\frac{\mathrm{b}}{\sin \theta}
\end{gathered}
$$

The radius of curvature of the ellipse at $B$ in the $A C A^{\prime}$ plane, is

$$
\rho=\frac{\mathrm{a}^{2}}{\mathrm{~b}}=\frac{1}{\mathrm{~b}} \cdot \frac{\mathrm{b}^{2}}{\sin ^{2} \theta}=\frac{\mathrm{b}}{\sin ^{2} \theta}=\frac{\mathrm{r}}{\sin ^{2} \theta}
$$

for $b=r$ the radius of the cylinder.

$$
\begin{aligned}
& \text { But } \mathrm{C}=\frac{1000}{\mathrm{f}^{\prime}}=\frac{1000(\mu-1)}{-\mathrm{r}} \text { in millimetres; } \\
& \mathrm{C}_{\theta}=\frac{1000(\mu-1)}{-\rho}=\frac{1000(\mu-1)}{-\mathrm{r}} \sin ^{2} \theta \text { in millimetres }
\end{aligned}
$$

(1) $\mathrm{C}_{\theta}=\mathrm{C} \sin ^{2} \theta$ in dioptres.

Consequently if $\theta$ be the angle of the plane axis with the meridian considered, the power $\mathrm{C}_{\theta}$ in that meridian is always $C \sin ^{2} \theta$.

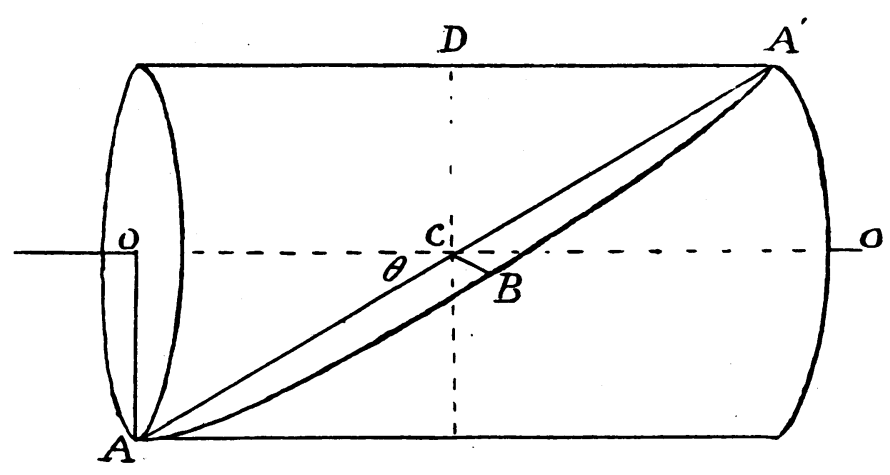

FIG. 2.

As the above reasoning was based on the fact that every oblique section of a cylindrical lens has an elliptical contour, it may be urged that I have no right to treat an ellipsoidal surface as a spherical surface. This would be a perfectly just criticism if the whole aperture of the lens were available, but the pupil of the eye only engages a very small area, of about $2 \mathrm{~mm}$. radius, rear the geometrical centre of the lens. As the radius of curvature of the cylindrical lens is usually more than $250 \mathrm{~mm}$., the small area engaged is indistinguishable from a spherical surface. It is true that when during eccentric vision peripheral parts of ordinary spherocylinders are employed, neitber the astigmatism nor the heterophoria is accurately corrected. This rarely causes much inconvenience, and if it does, the patient learns to avoid it by moving his head and not his eyes, so as always to use the central parts of his lenses.

Returning now to the application of the formula (1), if AC in Fig 1. represent the direction of the plane axis' of $\mathrm{a}-4 \mathrm{D}$ cylinder, the angle $\mathrm{OAC}(\theta)$ would be represented on the trial frame by $150^{\circ}$, and the angle between $\mathrm{AC}$ and the vertical meridian would be $\theta-90$. Consequently the effective power of the $-4 \mathrm{D}$ cyl. ax. 
would be $-4 \sin ^{2} \theta$ in the horizontal meridian, and $-4 \sin ^{2}\left(\theta-90^{\circ}\right)$ or $-4 \cos ^{2} \theta$ in the vertical meridian. Of course, if $\theta=150^{\circ}$ it is simple enough.

In the horizontal meridian $-4 \sin ^{2} 150^{\circ}=-4\left(\frac{1}{2}\right)^{2}=-1 \mathrm{D}$, in the vertical meridian $-4 \cos ^{2} 150^{\circ}=-4\left(\frac{\sqrt{ } 3}{2}\right)^{2}=-3 \mathrm{D}$.

This can be easily verified with a lens measure, if it be placed at $30^{\circ}$ with the plane axis of a $4 \mathrm{D}$ cylinder the reading is 1 , and if placed at $60^{\circ}$ the reading is 3.

In most cases, however, it is very troublesome to find the squares of sines and cosines, but the following simple trigonometrical formulae enable us to read off their values directly from the ordinary tables.

$$
\begin{aligned}
& \sin ^{2} \theta=\frac{1}{2}(1-\cos 2 \theta)=\frac{1}{2} \text { vers } 2 \theta \\
& \cos ^{2} \theta=\frac{1}{2}(1+\cos 2 \theta)
\end{aligned}
$$

Suppose now that we wish to combine the effect of a $1 \bar{\nabla}$ edge up and a $1.75 \nabla$ edge out with a spherocylinder of $-5 \mathrm{D}-4 \mathrm{D}$ cyl. ax. $15^{\circ}$, the procedure in this troublesome case would be as follows.

In the horizontal plane the effective power would be

$-5-4 \sin ^{2} 15^{\circ}=-5-2\left(1-\cos 30^{\circ}\right)=-5-2 \operatorname{vers} 30^{\circ}=-5.268 \mathrm{D}$.

In the vertical plane the power would be $-5-2\left(1+\cos 30^{\circ}\right)=-5-2(1 \cdot 866)=-8.732 \mathrm{D}$.

In the vertical plane $1 \nabla$ edge up is required, so that the appropriate vertical decentration $\mathrm{v}=\frac{10}{-8.732}=-1.145 \mathrm{~mm}$.

In the horizontal plane $1.75 \nabla$ edge out is required, so the horizontal decentration $\mathrm{h}=\frac{17 \cdot 5}{-5.268}=-3.322 \mathrm{~mm}$.

An oblique decentration $l \mathrm{~mm}$. in length at angle $\alpha$ will give the prismatic effect required, where $1=\sqrt{\mathrm{v}^{3}+\mathrm{h}^{2}}$ and $\tan \alpha=\frac{\mathrm{v}}{\mathrm{h}}$

$$
\begin{aligned}
& 1=\sqrt{1 \cdot 311+11.036}=3.514 \mathrm{~mm} . \\
& \tan \alpha=\frac{1 \cdot 145}{3 \cdot 322}=\cdot 3447 \quad \therefore \alpha=19^{\circ}
\end{aligned}
$$

The lens should therefore be decentred $3.5 \mathrm{~mm}$. outwards and upwards $19^{\circ}$ from the horizontal.

With the help of logarithms or a slide rule the above calculations can be very quickly made, as we can rarely prescribe prisms with greater accuracy than $\cdot 5 \nabla$, and no optician can be expected to decentre a lens in tenths of millimetres, or to measure its obliquity in anything finer than degrees. This example will clearly show the method of decentring spherocylinders. 


\section{Two Oblique Cylinders}

Two cylinders $C$ and $C_{2}$ set at angles $\alpha$ and $B$ are equivalent to a spherical lens of power $\mathrm{D}$ combined with a cylinder $\mathrm{C}_{3}$ set at an angle $\gamma$. The methods which have been given for determining $D$, $\mathrm{C}_{3}$ and $\gamma$ are usually very tedious, and some of them are inaccurate.

This is a short and simple method.

It has been shown that a cylinder of power $C$ if set with axis at $a$ exerts in the horizontal plane an effective power

$$
\mathrm{C} a=\frac{\mathrm{C}}{2}(1-\cos 2 \alpha)
$$

and in the plane at $45^{\circ}$ an effective power

$$
\mathrm{C}_{a-45^{\circ}}=\frac{\mathrm{C}}{2}\left(1-\cos 2 \overline{a-45^{\circ}}\right)=\frac{\mathrm{C}}{2}(1-\sin 2 \alpha)
$$

Hence the effective power in the horizontal plane of $C_{1}$ at $a$ and $\mathrm{C}_{2}$ at $\beta$ is given by

(A) $\frac{\mathrm{C}_{1}}{2}(1-\cos 2 \alpha)+\frac{\mathrm{C}}{2}(1-\cos 2 \beta)=\frac{\mathrm{C}}{2}(1-\cos 2 \gamma)+\mathrm{D}$

and the power in the plane at $45^{\circ}$ is given by

(B) $\frac{\mathrm{C}_{1}}{2}(1-\sin 2 \alpha)+\frac{\mathrm{C}_{2}}{2}(1-\sin 2 \beta)=\frac{\mathrm{C}}{2}(1-\sin 2 \gamma)+\mathrm{D}$.

It is clear that whatever be the plane considered the relation

(a) $\frac{\mathrm{C}}{2}+\frac{\mathrm{C}_{2}}{2}=\frac{\mathrm{C}_{3}}{2}+\mathrm{D}$, or $\mathrm{D}=\frac{\mathrm{C}_{1}+\mathrm{C}_{2}-\mathrm{C}_{3}}{2}$ holds.

On subtracting (a) from (A) and (B) we obtain

(I) $\mathrm{C}_{1} \cos 2 \alpha+\mathrm{C}_{2} \cos 2 \beta=\mathrm{C}_{3} \cos 2 \gamma$.

(II) $\mathrm{C}_{1} \sin 2 \alpha+\mathrm{C}_{2} \sin 2 \beta=\mathrm{C}_{3} \sin 2 \gamma$

The shortest method is first to find $2 \gamma$. On dividing (II) by the equals in (I) two values of $2 \gamma$ between 0 and $360^{\circ}$ are obtained, and therefore two values of $\gamma$ between 0 and $180^{\circ}$.

(b) $\tan 2 \gamma=\frac{\mathrm{C}_{1} \sin 2 \alpha+\mathrm{C}_{2} \sin 2 \beta}{\mathrm{C}_{1} \cos 2 \alpha+\mathrm{C}_{2} \cos 2 \beta}$

(c) Finally $\mathrm{C}=\frac{\mathrm{C}_{1} \cos 2 \alpha+\mathrm{C}_{2} \cos 2 \beta}{\cos 2 \gamma}$

which gives a positive or negative value of $\mathrm{C}_{3}$ according to the sign of $\cos 2 \gamma$.

Example: Find the resultant effect of a-3D cyl. ax. $15^{\circ}$ and a $+4 \mathrm{D}$ cyl. ax. $60^{\circ}$.

Here $\beta-\alpha=45^{\circ}$, and it is very tempting to find $C_{3}$ and D first by squaring and adding I and II, but we shall then get into difficulties about the values of $\gamma$. In order to show the advantage of finding $2 \gamma$ from $\tan 2 \gamma$, we will begin by squaring and adding (I) and (II) in order to find $\mathrm{C}_{3}$ : 


$$
\begin{aligned}
& \quad \mathrm{C}^{2}+2 \mathrm{C}_{1} \mathrm{C}_{2}(\cos 2 \alpha \cos 2 \beta+\sin 2 \alpha \sin 2 \beta)+\mathrm{C}_{2}^{2}=\mathrm{C}_{3}^{2} \\
& \text { or } \mathrm{C}_{1}^{2}+2 \mathrm{C}_{1} \mathrm{C}_{2} \cos 2(\alpha-\beta)+\mathrm{C}_{2}^{2}=\mathrm{C}_{8}^{2} \\
& \text { here } 9-24 \cos \left(-90^{\circ}\right)+16=\mathrm{C}_{3}^{2}=25 \\
& \therefore \mathrm{C}_{3}= \pm 5 \text {, and by (a) } \mathrm{D}=\frac{1}{2}(-3+4 \mp 5)=-2 \text { or }+3
\end{aligned}
$$

The solution is then $-2 \mathrm{D}$ sph. $+5 \mathrm{D}$ cyl. or $+3 \mathrm{D} \mathrm{sph} .-5 \mathrm{D}$ cyl., but if we attempt to find $\boldsymbol{\gamma}$ from either (I) or (II) we shall obtain two values of $2 \gamma$ if $\mathrm{C}_{3}$ is +5 , and two other values if $\mathrm{C}_{3}$ is -5 . We must use (b) and find $\tan 2 \gamma$.

$$
\begin{gathered}
\tan 2 \gamma=\frac{-3 \sin 30^{\circ}+4 \sin 120^{\circ}}{-3 \cos 30^{\circ}+4 \cos 120^{\circ}}=\frac{-1 \cdot 5+2 \sqrt{ } 3}{-1.5 \sqrt{ } 3-2}=\tan \left(n \pi-23^{\circ} 8^{\prime}\right) \\
\therefore 2 \gamma=156^{\circ} 52^{\prime} \text { or } 336^{\circ} 52^{\prime} \text { and } \gamma=78^{\circ} 26^{\prime} \text { or } 168^{\circ} 26^{\prime}
\end{gathered}
$$

As the denominator is negative, from (c) it is seen that $\cos 2 \gamma$ must be negative if $\mathrm{C}_{3}$ is positive and vice versa. The combination then is equivalent to either

- $2 \mathrm{D}$ sph.+5 D cyl. ax. $78^{\circ} 26^{\prime}$ or $+3 \mathrm{D}$ sph. $-5 \mathrm{D}$ cyl. ax. $168^{\circ} 26^{\prime}$

This, however, is not a mere mathematical exercise, but it explains a phenomenon that all careful retinoscopists must have observed, which has puzzled me for the last twenty years. Suppose the trues correction for a patient to be $+3+4$ cyl. ax. $85^{\circ}$, and when doinge the retinoscopy the surgeon has corrected the meridian of leasto refraction with a convex lens and that on adding a convex lens of ? +3.5 $\mathrm{D}$ he has nearly corrected the refraction in the meridian about $90^{\circ}$. He should then substitute a +3.5 cylinder for the sphere as this will give him the best objective means of determining the position of the axis. Suppose that he puts it at $90^{\circ}$. On rotating the mirror he will then find that the light does not move horizontally but obliquely at about $150^{\circ}$ as indicated on the trial frame. This shows at once that the cylinder is not set correctly, and that it requires rotating clockwise. It will require a rotation of exactly $5^{\circ}$ for the movement of the light to occur at right angles to the plane axis of the cylinder, and this is the final objective test of the correct position of the cylinder. As the obliquity of the shadow magnifies the error in position of the cylinder, this is seen to be a very exact method of determining the correct axis of the cylinder.

In the above case we may suppose the eye to be of astigmatism $-4 \mathrm{D}$ at $85^{\circ}$ and, the hypermetropia having been already corrected, we have only to find the resultant effect of -4 cyl. ax. $85^{\circ}$ and +3.5 cyl. ax. $90^{\circ}$.

By (b) $\tan 2 \gamma=\frac{-4 \sin 170^{\circ}+3.5 \sin 180^{\circ}}{-4 \cos 170^{\circ}+3.5 \cos 180^{\circ}}=\frac{-4 \sin 10^{\circ}}{+4 \cos 10^{\circ}-3.5}$ $=\frac{-\cdot 6945928}{+\cdot 4392312} \therefore 2 \gamma=\mathrm{n} \pi-57^{\circ} 42^{\prime}$, nearly, i.e., $122^{\circ} 18^{\prime}$ or $302^{\circ} 18^{\prime}$ 
As the astigmatism is still undercorrected, $\mathrm{C}_{3}$ is negative, and so $\cos 2 \gamma$ in (c) must be negative, i.e., $\cos 2 \gamma=\cos 122^{\circ} 18^{\prime}$ or $\gamma=61^{\circ} 9^{\prime}$ but $90^{\circ}-61^{\circ}=29^{\circ}$ and we must turn the cylinder $5^{\circ}$ clockwise, or practically about $\frac{1}{6}$ of the error suggested by the obliquity of the movement of the light. It will be found in this way that when the error in the position of the axis of the cylinder is $5^{\circ}$, if the error in the correction of the astigmatism be $1 \mathrm{D}$, whether in excess or defect, the obliquity of the movement of the light will magnify the real fault in position about four times, but if the error in the astigmatic correction be $0.5 \mathrm{D}$, the magnification of the fault in position will be about six times.

\section{ANNOTATIONS}

\section{Ship Lighting}

The Illuminating Engineering Society are gradually getting a collection of valuable papers on all lighting problems written by practical experts in their own line. One of the latest contributions appears in their Journal for May of this year and deals with the question of ship lighting in relation to safety, comfort and efficiency. The writer was Mr. W. J. Jones and in the subsequent discussion representatives of the Navy and mercantile marine took part. The problem of safe and efficient lighting of ships is a very special one with difficulties of its own. There seems to be very little literature on the subject. The Board of Trade regulations leave considerable latitude, and there seems to be but little effort at any system of standardization of illumination or fittings. The paper dealt mainly with technical details such as the nature of the lamps and fittings and the arrangements for indicating the failure of important lamps like sidelights, but several matters such as the efficient lighting of charts, etc., also received attention. It was suggested that as charts were made and examined by daylight some system of artificial daylight coming from below might bc better than the usual system. Captain Nicholson pointed out that the navigating officer in bad weather or coastal navigation avoided the chart room at night as far as possible, owing to the bright light preventing him from seeing anything for some time after coming out. He had found that by closing one eye, and keeping it closed while in the chart room, he was not affected in this way and could see quite well on coming out. Another interesting point noted by Commander Waller was that the Navy used twice as much light in the starboard light as in the port one on account of the greater absorption that took place in the green glass. 